\title{
ALTERATIONS IN SIGNAL DYNAMICS DURING REGULATION OF AIRWAY REFLEX RESPONSE
}

Regulatory airway mechanisms were studied on the basis of phrenic nerve activity which was compared during eupnea and aspiration reflex (AspR). Due to changing degrees of signal stationarity, discrete wavelet transformation enabling multiresolution analysis was performed, followed by calculation of wavelet energy and wavelet entropy parameters.

The higher rate of wavelet energy was detected in lower frequency components during AspR in contrast to eupnea. Moreover, quiet inspiration is characterized by higher wavelet entropy comparing to the AspR, indicating the probability of its random character and varying degree of system disorder during transient between different respiratory responses.

Key words: biosignal processing, respiratory control, reflex, wavelet energy, entropy

\section{Introduction}

Since the early days of neuroscience research, understanding the regulation of physiological processes has become a fully multidisciplinary task, highly dependent on the usage of principles from mathematics, physics and computer science in many aspects of view. As a question resulting from the information theory, regulation on respiratory reflex responses is conditioned by a degree of synergy related to complex multilevel and multibehavioral neuronal networks in the rostral ventrolateral medulla. Generation of respiratory activity and its modulation towards the organism's requirements remains still not fully explained.

Information resulting from the control mechanisms is carried through nerves and muscles to achieve desired response and expressed in neurogram qualities, including timing, intensity, frequency composition and many others. Thus, the principal thesis of this approach is to suggest an optimal processing method considering fundamental irregular nature of biological signals.

Electrical signals resulting from different airway reflex responses have been traditionally analyzed via simple frequency analysis derived from the Fourier transformation. Power spectral analysis proved differences presented in power spectrum during eupnea (quiet inspiration) and other forms of inspiratory activity [1, 2]. However, Fourier transformation has many limitations in the case of electrophysiological data analysis [3], thus method involved in activity determination both in time and frequency domain appears to be necessary [4].

The intent of this work is to extract differences in phrenic neurogram between normal quiet inspiration and aspiration reflex
(AspR) - strong inspiratory activity, without subsequent active expiration that could be, contrary to other airway defense reflexes, elicited in any phase of respiratory cycle and characterized by ability to interrupt severe functional disorders [5]. Thus, this specific reflex response could probably represent a model of the brainstem neuronal network reconfiguration [6].

In this paper, the motor behavior of different respiratory responses was described as a function of energetic distribution over time - frequency domain. The action potentials recorded from respiratory nerve output were analyzed during quiet inspiration and aspiration reflex to describe changes of dynamics related to its spectral features. Discrete wavelet transformation allowing multiresolution analysis was used to decompose original phrenic nerve recording into particular scale rates enabling determination of wavelet energy relating to separate frequency components. Moreover, the wavelet entropy parameter was calculated to illustrate a degree of system order/disorder, reflecting merits of respiratory activity generation and control.

\section{Material and methods}

\subsection{Data recording and preprocessing}

Phrenic nerve activity was recorded from five adult cats of both genders anesthetized by pentobarbital (initial intraperitoneal dose (35-40) mg. $\left.\mathrm{kg}^{-1}\right)$. Aspiration reflex was elicited mechanically by touching the nasopharyngeal mucosa. An elastic nylon fibre $(0.4 \mathrm{~mm})$ was introduced through a pharyngostomy to elicit appropriate reflex. The electrical activity from right phrenic nerve was scanned using bipolar silver electrode which was connected to

\footnotetext{
* Juliana Knocikova

Department of Physics, Faculty of Electrical Engineering, University of Zilina, Slovakia, E-mail: knocikova@fel.uniza.sk
} 

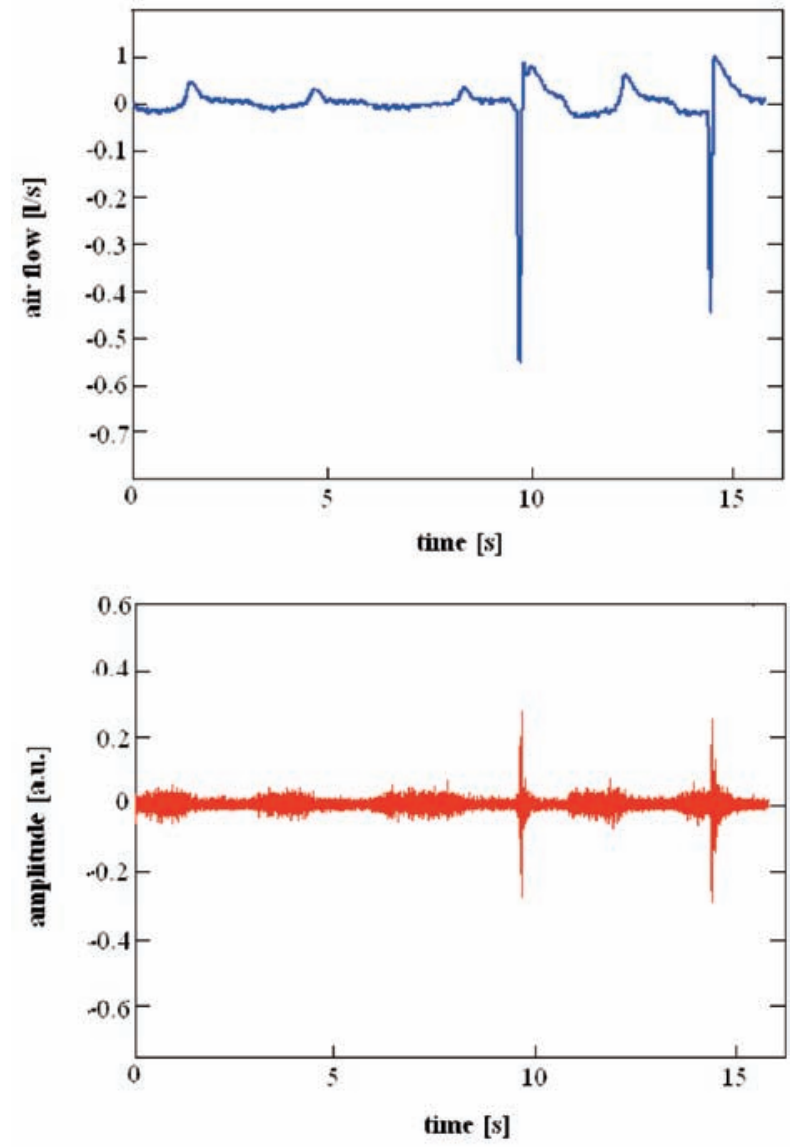

Fig. 1 Air flow and phrenic nerve recordings during quiet inspiration bursts followed by short strong inspiratory activity of AspR.

a low-noise amplifier. Neurograms were digitalized and sampled using minimum sampling rate of $2 \mathrm{kHz}$. These procedures have been described previously in details $[2,7]$.
Nerve recordings (Fig. 1.) were pass-band filtered and frequency components from interval $(30-1000) \mathrm{Hz}$ were analysed. $50 \mathrm{~Hz}$ frequency was cut off through a stop-band elliptic filter to reduce impact of power supply noise on energy of correspondent frequency bands. The wavelet energy and entropy were calculated in 10 eupneic and 10 AspR bursts, only the single bursts of electrical phrenic nerve activity were considered.

\subsection{Data analysis}

\section{Discrete Wavelet transformation (DWT)}

The basic principle of the discrete wavelet function consists in signal decomposition into set of wavelets, small oscillations with limited duration and mean value equal to zero. DWT exploits a wavelet basis functions and exhibits zero redundancy. DWT coefficients could be calculated using the following formula:

$$
\psi_{m, n}(p)=\frac{1}{\sqrt{s_{0}^{m}}} \psi\left(\frac{t-n p_{0} s_{0}^{m}}{s_{0}^{m}}\right),
$$

where $m, n \in Z$.

Integers $m$ and $n$ control the wavelet dilation and/or translation; $s_{0}$ is a fixed dilation step parameter set at a value greater than 1 and $p_{0}$ is the location parameter set at a value greater than zero. Usually, a common choice for discrete wavelet parameters $s_{0}$ and $p_{0}$ is 2 , known as the dyadic grid arrangement.

During DWT, lower frequencies of original signal (approximate coefficients) are separated from higher frequencies (detail coefficients) via low and high pass filtration. Number of data is then doubled. Approximate coefficients could be filtered again (Fig. 2.) to obtain approximate and detail coefficients at a higher frame (Fig. 3.) resulting in scale accommodation (scale $2^{n}$ for $n$-frame). In this study, decomposition at 8 level of approximate and detail coefficients was used. During this procedure, daubechies 3 was employed as a mother wavelet.

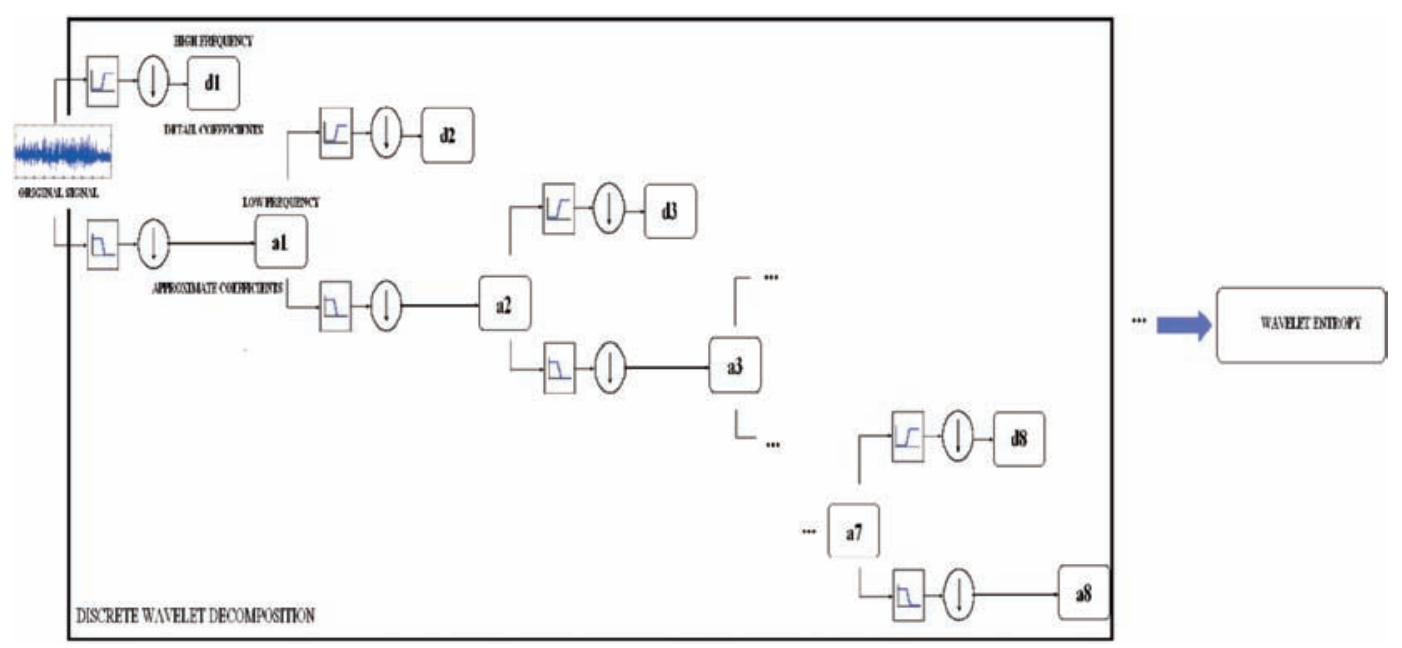

Fig. 2 Discrete wavelet decomposition of original phrenic signal at 8 level approximate and detail coefficients 

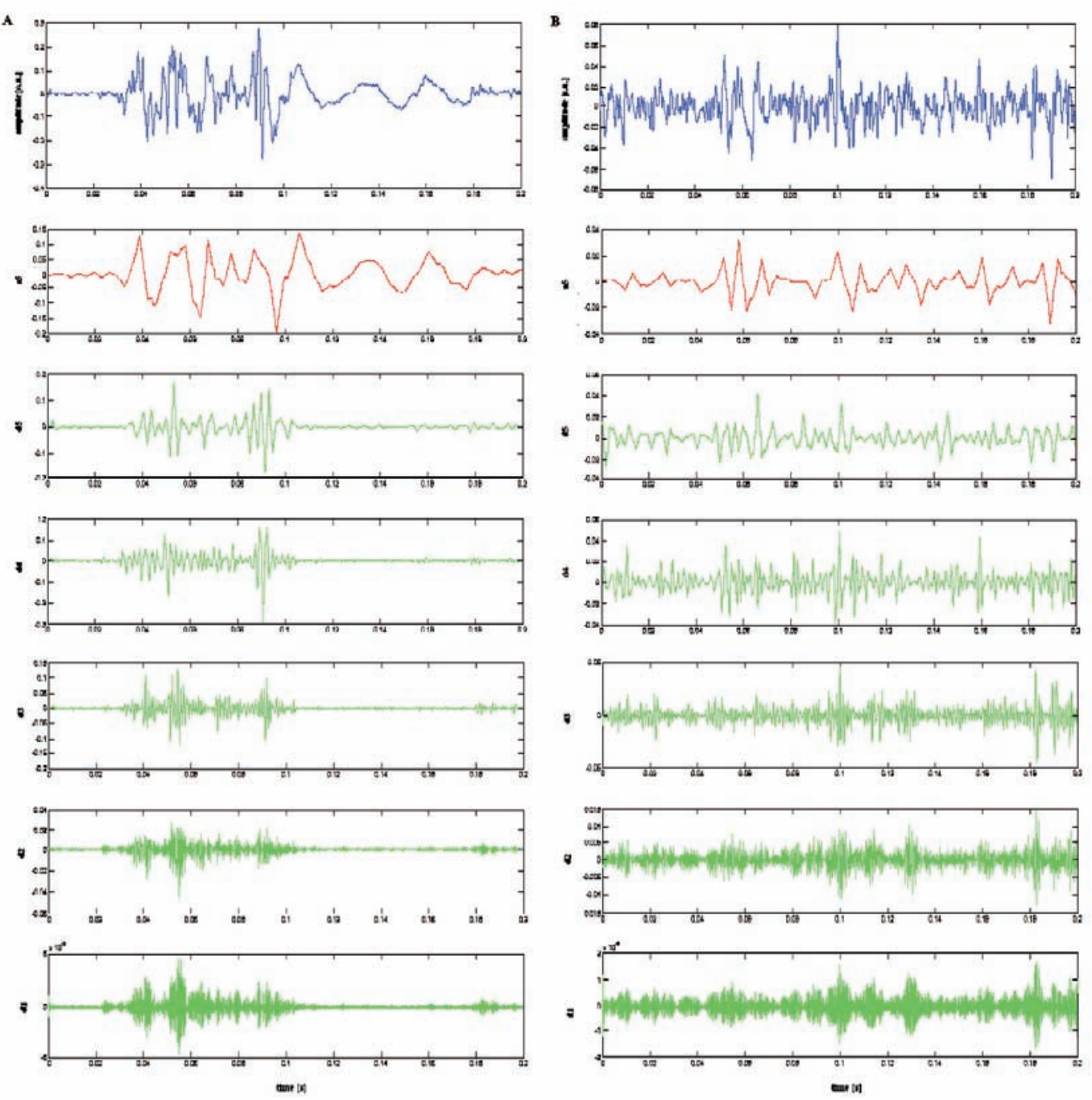

Fig. 3 Result of discrete wavelet decomposition of original activity (blue) during AspR (A) and eupnea (B). $a$ - approximate coefficients, $d$ - detail coefficients

Daubechies 3 is often used in biosignal analysis for detection of transient oscillations. Daubechies wavelet showed profitable properties for pattern classification during analysis of brain function [8]. Dynamical changes of the phrenic nerve electrical activity in time and frequency analyzed in this study probably reflect information about specific neuronal components involved in the generation of inspiratory activity.

\section{Wavelet energy and wavelet entropy}

For wavelet coefficients $c_{s}(k)$ at each resolution level s, the wavelet energy at each time sample $\mathrm{k}$ is obtained as:

$$
E(k)=\sum_{s=1}^{s}\left|c_{s}(k)\right|^{2}
$$

The total wavelet energy is then computed as:

$$
E_{T O T}=\sum_{s=1}^{s} \sum_{k=1}^{N}\left|C_{s}(k)\right|^{2}=\sum_{s} E_{s}
$$

Relative wavelet energy parameter quantifying the distribution of energy over appropriate scales is:

$$
P_{s}=\frac{E_{s}}{E_{T O T}}
$$

Thus, the wavelet entropy is defined as:

$$
W E=-\sum_{s=1}^{s} p_{s} \log _{2}\left[p_{s}\right]
$$

\subsection{Statistics}

All analyzed parameters were compared between two groups - phrenic bursts during aspiration reflex and quiet inspiration. 10 eupneic and $10 \mathrm{AspR}$ bursts of phrenic electrical activity were 
considered. The statistical evaluation of data was realized through unpaired t- test and Mann-Whitney test. Suitable test (parametric or nonparametric) has been chosen due to the fulfilment of condition for normal data distribution. Differences were considered significant for $p<0.05$.

\section{Results}

Discrete wavelet transformation disclosed significant differences in time - frequency energetic distribution between both behaviors. During eupnea, the energy was distributed uniformly over the burst duration. However, energy of AspR was cumulated especially around the maximal intensity interval $(p<0.05)$

Prominent energetic differences were detected in frequency domain. While AspR was characterized by strong energetic accumulation at lower frequencies corresponding to the approximate coefficients ( $p<0.0001$, Fig. 3 ), the eupneic activity showed increasing wavelet energy at higher frequency components $(p=0.0022)$, corresponding to interval $(125-250) \mathrm{Hz}$.

Parameter wavelet entropy showed significant differences between analyzed groups (Fig. 4.). AspR resulted in its decreasing $(p=0.0023)$.

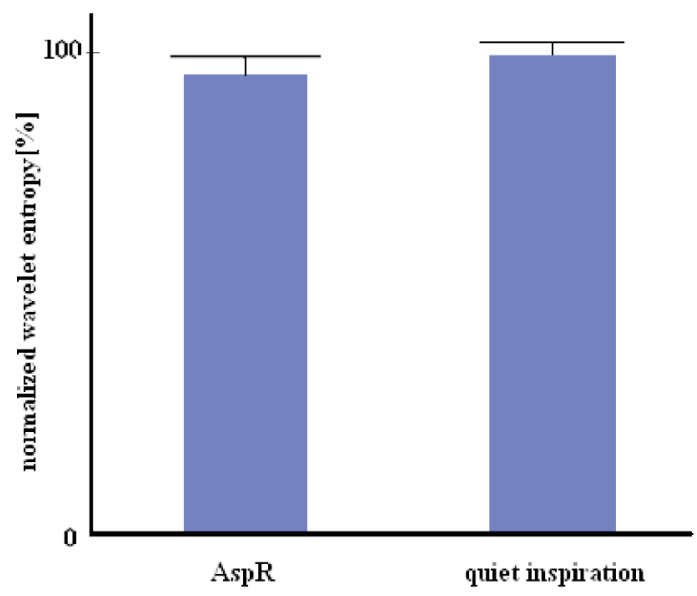

Fig. 4 The wavelet entropy measure normalized to averaged entropy of quiet breathing (mean \pm standard deviation). AspR results in wavelet entropy decreasing $(p=0.0023)$.

\section{Discussion}

The most important finding of this study is that the time - frequency distribution of energy derived from phrenic nerve activity shows differences between two inspiratory activities. Lower frequencies are markedly involved in energy creation during aspiration reflex under comparison with eupnea. Moreover, eupnea results in increasing of wavelet entropy parameter.
As stated in Material and methods, we considered neural activities within the range of $(30-1000) \mathrm{Hz}$. With regard to analysis of the respiratory nerve outputs, the most interesting frequency range is above $30 \mathrm{~Hz}$, including the high frequency oscillations in cat. These activities were described as the most distinguished signs of respiratory neurogram spectra, significantly contributing to the total power of inspiration [9].

In every sense, there is a lack of information regarding to spectral characteristics in AspR and their comparison with other defensive airway reflexes. Moreover, frequency components of respiratory outputs depend markedly on different experimental conditions and methodical approaches and major part of papers deals with different animal model, type of anesthesia or filtering methods significantly affecting the signal spectra. Previous studies of the cat phrenic nerve activity confirmed markedly different power spectra in AspR and eupnoe using Short Fast Fourier Transform [2]. This important identification was based on detection of typical high frequency oscillations and their position in power spectra as a solitary spectral characteristics. Therefore, analysis based on determination of wavelet entropy and time - frequency distribution of whole energy involved in phrenic bursts provides complementary findings in the field of spectral behavior of defensive airway reflexes.

The method of wavelet transformation, widely used for analysis of nonstationary and fast transient signals, represents effective alternative towards Fourier transformation. The most important advantage of the wavelet transformation consists in continuous changes of the mother wavelet (an alternative of window in Fast Fourier Transformation) according to its scale and time position. With regard to adapting length of the weighing function, wavelet transformation provides an adequate time and frequency localization of signal features by using short windows at higher frequencies and long windows at lower frequencies. An optimal time-frequency resolution [10] is reached by scaling and translation of mother wavelet function - a short duration oscillatory curve with zero-mean value [11]. Consequently, analyzed signal is decomposed into modified version of mother wavelet [12]. Therefore, lower frequencies (slow oscillations) are better distinguishable in frequency domain while higher frequencies (fast oscillations) are a better time distinguishable because of narrower window function [13].

Relative energy associated with different frequency segments of neurogram exhibits significant differences in analyzed recordings. Approximate coefficients representing lower frequency components are dominant in creation phrenic signal during aspiration reflex. Similar findings were observed in adult decerebrate rats [14] during gasp - aborted breath, accompanied by strange vocalizations and muscles twitching. Loss in high frequency oscillations could suggest possible reconfiguration of the respiratory network when switching from eupnea. Relationships between aspiration reflex and gasping have been previously studied because of their similar manifestation and possible implications in clinical practice, especially potential for resuscitation effect [6]. Presented method of wavelet energy computation acknowledged other behavioral similarities, including distribution of energy over different scale/ 
frequency segments related to phrenic neurogram in aspiration reflex and gasping development.

Wavelet entropy is a measure estimated by the wavelet coefficients to provide quantitative information about the order/complexity of analyzed signals [15]. As a method of time - series processing, entropy can describe dynamical cause associated with manifestation of different neurogram stages. Higher wavelet entropy value detected in eupneic activity reflects higher probability of system disorder comparing to AspR. From this point of view, quiet breathing sounds like more random, irregular behavior. Probably, the total wavelet entropy may reflect synchrony level of neural firing [16]. Other investigators suppose that entropy could be an effective tool for measuring diffusion energy over neurons or degree of synaptic activity.

Decreased signal complexity during coughing and swallowing reflex in contrast to eupnea was investigated on the basis of the phrenic approximate entropy determination [17]. Similarly, implication of wavelet entropy analysis in this study certified random eupneic mode. Thus, reduced signal complexity probably results from synchronous activity of a homogeneous group of neurons during airway reflex response.

\section{Conclusion}

An important conclusion derived from this study is a presence of different time - frequency distribution of energy related to both analyzed respiratory behaviors. Lower wavelet entropy is resulting from transient from eupneic activity to aspiration reflex. This decreasing could be explained as a changing degree of neural firing synchronization. Finally, these findings indicate specific processes at a level of central integration of aspiration reflex with potential impact on understanding the running of respiratory control mechanisms.

\section{Acknowledgement}

The author would like to thank to all co-workers (Department of Med. Biophysics, Comenius University, Slovakia) for participating in data recording.

\section{References}

[1] YANAURA, S., KAMEI, J., GOTO, K., HOSOKAWA, T., MISAWA, M., HUKUHARA, T.: Analysis of Efferent Discharges of the Phrenic Nerve During the Cough Reflex, Jpn J Pharmacol, 1982, 32: 795-801.

[2] TOMORI, Z., FUNG, L. M., DONIC, V., DONICOVA V., St JOHN W. M.: Power Spectral Analysis of Respiratory Responses to Pharyngeal Stimulation in Cats: Comparisons with Eupnoea and Gasping. J Physiol, 1995, 485: 551-559.

[3] KNOCIKOVA, J., POLIACEK, I., CAP, I., BARANI, H., JAKUS, J.: Wavelet Analysis of Electrical Activities from Respiratory Muscles during Coughing and Sneezing in Anaesthetized Rabbits. Acta Vet Brno, 2009, 78: 387-397.

[4] O'NEAL, M.H., SPIEGEL, E. T., CON, K. H., SOLOMON, I. C.: Time-frequency Representation of Inspiratory Motor Output in Anesthetized C57BL/6 Mice in Vivo. J Neurophysiol. 2005, 93: 1762-1775.

[5] TOMORI, Z.: In: Cough and Other Respiratory Reflexes, Vol. 12, Ed. BASEL, H.H. Karger, p. 356, 1979.

[6] TOMORI, Z., BENACKA, R., DONIC, V., TKACOVA, R.: Reversal of Apnoea by Aspiration Reflex in Anaesthetized Cats. Eur Respir J, 1991, 4: 117-1125.

[7] TOMORI, Z., POLIACEK I., KNOCIKOVA, J., JAKUS, J., DONIC, V.: Distinct Generators of Aspiration and Expiration Reflexes, their Localizations, Mechanisms and Effects. ERS Congress. - Berlin: [s.n.]. 2008, p. 144

[8] KIM M. S., CHO Y. CH., BERDAKH A., SEO H. D.: Analysis of Brain Function and Classification of Sleep EEG using Daubechies Wavelet, Sensors and Materials, 2008, 20: 1-14.

[9] COHEN, M. I., SEE W. R., CHRISTAKOS C. N., SICA A. L.: High-frequency and Medium-frequency Components of Different Inspiratory Nerve Discharges and their Modification by Various Inputs. Brain Res, 1987, 417: 148-152.

[10] NAJMI, A. H., SADOWSKI, J.: The Continuous Wavelet Transform and Variable Resolution Time-frequency Analysis. The Johns Hopkins APL Technical Digest, 1997, 18: 134-140.

[11] DAUBECHIES, I.: The Wavelet Transform, Time-frequency Localization, and Signal Analysis. IEE Trans Info Theory, 1990, 36: 961-1005.

[12] GOUPILlAUD, P., GROSSMANN, A., MORLET, J.: Cycle-octave and Related Transforms in Seismic Signal Analysis. Geoexploration, 1984, 23: 85-102.

[13] MALLAT, S.: A Theory for Multiresolution Signal Decomposition: the Wavelet Representation. IEEE Trans Patt Anal Mach Intell, 1989, 2: 674-693.

[14] MARCHENKO, V., ROGERS, R. F.: Selective Loss of High-frequency Oscillations in Phrenic and Hypoglossal Activity in the Decerebrate Rat during Gasping. Am J Physiol Regul Integr Comp Physiol, 2006, 291: R1414-R1429.

[15] PINCUS, S. M., GOLDBERGER, A. L.: Physiological Time-series Analysis: What does Regularity Quantify? Am J Physiol, 1994, 266: H1643-H1656.

[16] ZHENG, X., SUN, M., TIAN, X.: Wavelet Entropy Analysis of Neural Spike Train. Congress on Image and Signal Processing, 2008, 1: $225-227$.

[17] DRAGOMIR, A., AKAY, Y., CURRAN, A. K., AKAY, M. Investigating the Complexity of Respiratory Patterns during the Laryngeal Chemoreflex. J Neuroeng Rehab, 2008, 5:17. 